\section{The effect of nano-silver packaging in increasing the shelf life of nuts: An in vitro model}

\author{
Hamidreza Tavakoli, ${ }^{1}$ \\ Hossein Rastegar, ${ }^{2,3}$ Mahdi Taherian, ${ }^{2}$ \\ Mohammad Samadi, ${ }^{4}$ Hossein Rostami ${ }^{1}$ \\ ${ }^{1}$ Health Research Center, Baqiyatallah \\ University of Medical Sciences, Tehran; \\ ${ }^{2}$ Food and Drug Research Institute, Iran \\ Food and Drug Administration, \\ Ministry of Health and Medical \\ Education, Tehran; ${ }^{3}$ Cosmetic Products \\ Research Center, Iran Food and Drug \\ Administration, Ministry of Health and \\ Medical Education, Tehran; ${ }^{4}$ Exercise \\ Physiology Research Center, \\ Baqiyatallah University of Medical \\ Sciences, Tehran, Iran
}

\begin{abstract}
Nano packaging is currently one of the most important topics in food packaging technologies. The aim of the application of this technology in food packaging is increasing shelf life of foods by preventing internal and external corruption and microbial contaminations. Use of silver nanoparticles in food packaging has recently attracted much attention. The aim of this study was to investigate the effect of nano-silver packaging in increasing the shelf life packages of nuts in an In vitro model. In this experimental study, the effects of different nano-silver concentrations $(0,1,2$ and 3 percent) on biological and chemical properties of 432 samples of nuts including walnuts, hazelnuts, almonds and pistachios were evaluated during $0,3,6,9,12,15,18$, 21 and 24 months. In most samples, different concentrations of nano-silver (1, 2 and 3 $\%)$ significantly reduced total microbial count, mold and coliform counts compared to control group and the 3\% nano-silver concentration was more effective than other concentrations $(\mathrm{P}<0.05)$. Moreover, using this packaging yielded an antioxidant effect especially when $2 \%$ and $3 \%$ nano-silver concentrations were used. Nano-silver also prevented growth of mold and so prevented aflatoxin production in all treatment groups. Results of chemical and biological tests showed that the silver nanoparticles had a significant effect on increasing the shelf life of nuts. The highest shelf life belonged to pistachios, almonds, hazelnuts and walnuts with 20,19, 18 and 18 months, respectively. The shelf life was associated with amount of silver nanoparticles. The highest antimi-
\end{abstract}

crobial activity was observed when 3\% nano-silver concentration was used in pistachios. The shelf life of control groups in similar storage conditions were calculated for an average of 13 months. In conclusion, the results of this study demonstrate the efficacy of nano-silver packing in increasing shelf life of nuts. Hence, use of nano-silver packaging in food industry, especially in food packaging is recommended.

\section{Introduction}

One of the main issues in food processing is the protection against foodborne diseases which still represents a global problem of public health (Morris Jr, 2011). Food packaging systems are recently under high investigation to lower risk of foodborne diseases. Nowadays, the use of nanomaterials in several fields is growing (Carbone et al., 2016) and also for food packaging, it is largely increased over the past decade (Bumbudsanpharoke and Ko, 2015). In addition to their higher health safety, materials produced by nanotechnology have lower price and higher quality (Rashidi et al., 2011). Metal nanoparticles with their potent antimicrobial properties are used as active packaging. Emerging metal nanoparticles with biocidal properties are $\mathrm{Cu}, \mathrm{Zn}$, $\mathrm{Au}, \mathrm{Ti}$, and Ag (Toker et al., 2013). Among them silver nanoparticles (AgNPs) demonstrated to have the most effective bactericidal properties against a wide range of pathogenic microorganisms, including bacteria, yeasts, fungi and viruses (Martinez-Abad et al., 2012). Thus, besides of increasing shelf life, this packaging system does not change the food physical characteristics (Emamifar et al., 2010).

Some previous studies have investigated the beneficial effect of nano-silver packaging on food shelf life and quality. Foroughi et al. in 2011 reported applying coating titanium oxide which covered with nano-silver on the packaging of meat products. Emamifar et al. (2010) applied nano-silver particle plus zinc oxide on the packaging of orange juice. Sattari et al. (2010) used particles of clay and silver for the packaging of volume bread. Antimicrobial effect of nanosilver packaging is investigated in some previous studies (Sánchez-Valdes et al., 2009; Incoronato et al., 2011). Sanchez et al. (2009) prepared a five-layer film as a substrate and a thin layer of the silver-polyethylene nanocomposite was deposited over it. This film had antimicrobial effects (Sánchez-Valdes et al., 2009). Incoronato et al. (2011) studied the influence of antimicrobial system containing nanoparticles of montmorillonite-silver for quality of cheese. This system increased shelf life of
Correspondence: Hossein Rastegar, Food and Drug Research Institute, Iran Food and Drug Administration, Ministry of Health and Medical Education, Tehran, Iran

Tel: +98 2166494428

E-mail: hossein.admrastegar@gmail.com

Key words: Nuts; Silver nano-particles; Chemical properties; Biological properties.

Conflict of interest: the authors declare no potential conflict of interest.

Funding: this study is funded by Health Research Center in Baqiyatallah University of Medical Sciences.

Acknowledgements: we appreciate the Health Research Center in Baqiyatallah, University of Medical Sciences for their financial support of this study.

Contributions: HT and HR made substantial contributions to conception and design of the study; HT, HR, MT and MS were involved in statistical analysis and interpretation of data; MT, MS and HR were responsible for in drafting the manuscript; HT and HR revised the manuscript critically; all authors have given their final approval of this version to be published.

Received for publication: 2 July 2017.

Revision received: 12 August 2017.

Accepted for publication: 4 September 2017

This work is licensed under a Creative Commons Attribution-NonCommercial 4.0 International License (CC BY-NC 4.0).

(C) Copyright H. Tavakoli et al., 2017

Licensee PAGEPress, Italy

Italian Journal of Food Safety 2017; 6:6874

doi:10.4081/ijfs.2017.6874

cheese by lowering the microbial count (Incoronato et al., 2011). The effect of the active coating containing nanoparticles of montmorillonite- silver on the shelf life of fresh carrot slices was investigated by Costa et al. (2011). They showed that using a combination of active coating and packaging cause preservation of carrots up to 70 days (Costa et al., 2011).

Although several previous studies have investigated the effect of nanoparticle coatings in reducing the number of total microbial count, none of them have studied the efficacy of nano-silver packaging in increasing the shelf-life and enhancing quality of nuts. Hence, this study aimed to assess the effect of using nano-silver packaging on shelf life, microbial and mold load and also peroxide value of food during 24 months of storage. 


\section{Materials and Methods}

This is an experimental and laboratory study aimed to evaluating effect of nano-silver particles embedded to polyethylene composites on microbiological and chemical status of long-term stored nuts. In this study, four types of commonly consumed nuts were selected (Pistachio, Walnut, Almond and Hazelnut). Four different concentrations $(0,1,2,3$ percent $)$ at different time intervals $(0,3,6,9,12,15,18,21,24$ months) were used to evaluate the effect of Silver nanoparticles according to national standard [standard NO. 8923-4 of Institute of Standards and Industrial Research of Iran (ISIRI)] with triplicate methods. The total sample size was calculated considering the number of nuts, different nano-silver concentrations, different time periods and the number of iterations, using the following formula:

$$
\mathrm{N}=4 \times 4 \times 9 \times 3=432
$$

The time intervals between the tests were determined according to national standard methods (standard NO. 8923-4 of ISIRI). Chemical and microbial control tests were done to determine the shelf life of products. For preparation of composite films, silver nanoparticles were chemically synthesized using $\mathrm{AgNO} 3$ as source materials and according to the detailed protocol reported by Darroudi et al. (2010). Polyethylenenano-silver composite films containing different silver concentrations were produced through the extrusion method. Fifty grams of four types of nuts, including pistachios, walnuts, almonds and hazelnuts was packaged in $0,1,2,3 \%$ nano-silver composite packages. To prevent oxygen entrance or leave, the packages were vacuumed by a vacuum pump and then was packaged by sealing machine and the containers were stored at room temperature. Non-vacuumed packages, without nanoparticles, were considered as control. Biological and chemical tests were done for stored samples at months $0,3,6,9,12,15,18,21$ and 24 .

\section{Microbial analysis}

For total count of microorganisms, $10 \mathrm{~g}$ sample was taken from each sample and was homogenized in a sterile bag with 90 $\mathrm{mL}$ of $0 / 1 \%$ peptone water in Bag mixer machine for 2 minutes. Then, serial dilutions in $9 \mathrm{ml}$ of diluent solution (from -1 to 5 according to the Iranian National Standard Organization procedures) were made. Counting on plates (in case of bacteria and mold between 30-300 and between 25-250 colonies, respectively) was based on international standards. From every dilution, $0.1 \mathrm{~mL}$ was transferred on BHI agar and cultured and then was incubated for 2448 hours at $35^{\circ} \mathrm{C}$. Then, number of colonies, average and total microbial count was calculated. Logarithmic method was used to report the results.

\section{Mold count}

From every dilution, $0.1 \mathrm{~mL}$ was transferred on DG18 agar and was spread using a glass rod. Then, plates were incubated for 3-5 days at $25^{\circ} \mathrm{C}$ and mold counts were calculated by counting number of colonies. The logarithmic method was used to report the results.

\section{Coliform count}

From every dilution, $1 \mathrm{~mL}$ was transferred on plate and mixed with $10 \mathrm{~mL}$ VRBA agar. Then plates were incubated for 18-24 hours at $35^{\circ} \mathrm{C}$ and were spread using a glass rod. Then they were incubated for 35 days at $35^{\circ} \mathrm{C}$. Coliform counts were calculated by counting the number of colonies.

\section{Measurement of peroxide value}

In order to assess oxidative corruption, peroxide value was measured by titration. $20 \mathrm{~g}$ sample with $100 \mathrm{~mL}$ of chloroform completely crushed in a mechanical stirrer and then the solution passed a filter paper containing dry sodium sulfate. A certain volume of the filtered solution transferred to a balloon and was dried in the oven and then the amount of fat was calculated.

To calculate peroxide value, $20 \mathrm{~mL}$ of the filtrate solution was poured in a balloon and $30 \mathrm{~mL}$ of acetic acid was added to it and then it was added to saturated solution of potassium iodine and was put in darkness for one minute. Then, $30 \mathrm{~mL}$ of distilled water was added and titration with sodium thiosulfate 0.1 solution was continued until a pale yellow color appeared. Next, few drops of starch indicator was added and the solution was shaken. The titration was continued until all released iodine from chloroform layer reacted with solution of sodium thiosulfate and the blue color disappeared. Peroxide value was calculated according to the following formula:

Peroxide $=(\mathrm{V} 1-\mathrm{V} 0) \mathrm{N} / \mathrm{m} \times 1000$

V0 $=$ The volume of sodium thiosulfate solution used in the control test according to $\mathrm{mL}$ $\mathrm{V} 1=$ The volume of sodium thiosulfate solution used in the sample test according to $\mathrm{mL}$ $\mathrm{m}=$ Fat mass in $20 \mathrm{~mL}$ of the filtrated solution according to $\mathrm{g}$

$\mathrm{N}=$ Normality of consumable sodium thiosulfate solution

$1000=$ Conversion coefficient gr to $\mathrm{kg}$ Unit Peroxide $=$ According to $\mathrm{mEq}$ per $\mathrm{kg}$ fat extracted

\section{Measurement of aflatoxin}

Measuring aflatoxin accomplished with high performance liquid chromatography and immunoaffinity column (HPLC) method. In this method, $5 \mathrm{~g} \mathrm{NaCl}, 120 \mathrm{~mL}$ pure methanol and $100 \mathrm{~mL} \mathrm{~N}$-hexane were added to $50 \mathrm{~g}$ of kneaded sample and then it was blended at high speed for 3 minutes. After the N-hexane phase separation of extract (20 mL of filtered extract), $120 \mathrm{~mL}$ distilled water was added and then was passed through filter paper. After immunoaffinity column reached laboratory temperature, $10 \mathrm{~mL}$ of PBS buffer was passed through that. Then $70 \mathrm{~mL}$ of the diluted extract from the column was passed at a rate of one drop per second. Then the column was washed with $15 \mathrm{~mL}$ of distilled water and was dried through gentle pressure for 5 to 10 seconds. In order to wash aflatoxin residues from columns, $1 / 5 \mathrm{~mL}$ of methanol (MeOH-HPLC) was added to column and were collected in a vial. Then $1 / 5$ $\mathrm{mL}$ of standard solution of HPLC was added to the vial and after vortexing up passing through the $45 \mu \mathrm{m}$ filter, 100 micro liters of the solution was injected to device. In order to quantitative estimation of aflatoxin, standard curve (Aflatoxin mix, Sigma Aldrich, St. Louis, MO, USA) of different

Table 1. The presence (+) or absence $(-)$ of aflatoxin in nuts packed with films containing different percentages of silver.

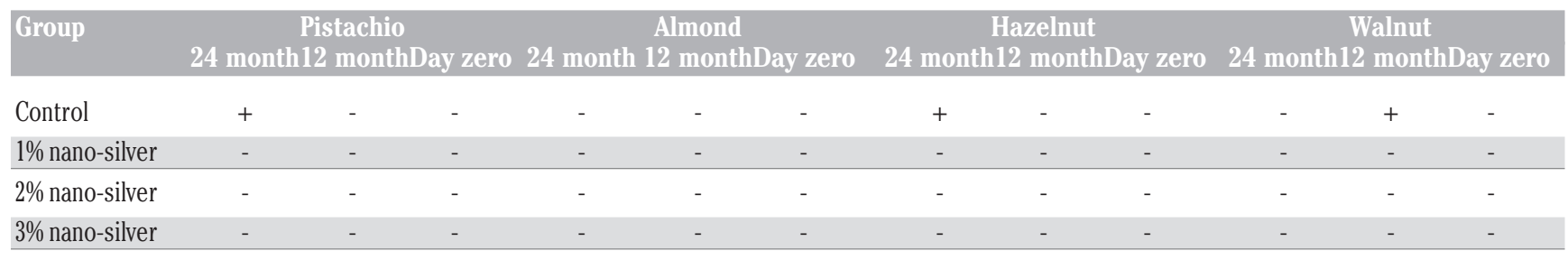


concentrations was used after considering the dilution coefficient. The data were converted to logarithm and were analyzed using a random statistical design. Duncan's test was used to compare means at a $5 \%$ level of significance.

\section{Statistical analysis}

To compare microbial count and also to analyze the amounts of peroxide value, oneway ANOVA and tukey's test were used. The differences among the mean values were found to be significant at $\mathrm{P} \leq 0.05$. SPSS V.20 and Excel software were used to draw graphs and table.

\section{Results}

\section{Microbial analysis}

\section{Total count}

Results of total microbial count of packed nuts in different concentrations of nano-silver are depicted in Figure 1. Regarding the Walnut in control group, a mild increase was observed in total microbial count during the storage time. The logarithm of total count in this group was increased from 3.6 at the beginning to the 5.6 at 18th month showing a nearly $2 \log$ increase. However, after 18 months, no significant change was observed in total count. In 1\% nano-silver group total microbial count was increased during storage time and no significant difference was observed compared to control group. By increasing nano-silver concentration to $2 \%, \log$ of total microbial count was increased from 3.6 at the beginning of storage time to 4.6 at 12 th month. At 3 percent concentration of nano-silver, no significant differences in total microbial count was observed at 24 months compared to beginning of the storage time (Figure 1A).

Results of total microbial counts of packed hazelnut in different concentrations of nano-silver are shown in Figure 1B. A mild increase in total microbial count of control group was evident during storage time. Log of total microbial count reached from 3 at the beginning to 5.5 at 18 th month which shows $2.5 \log$ increase in total count. However, after 18 months, no significant changes were observed in total count. Although total count increased in 1\% nanosilver group, it was significantly about $1 \mathrm{log}$ lower than control group. Increasing concentrations of nano-silver to $2 \%$ made no significant difference in total microbial count compared to $1 \%$ nano-silver group. In the $3 \%$ nano-silver group, total microbial count increased much lower than other groups and the final count was about $3.7 \mathrm{log}$ that showed lower than $1 \log$ increase com- pared to beginning of storage time.

Results of total microbial count of packed pistachio in different concentrations of nano-silver are shown in Figure 1C. It seems that this results are similar to that of walnut. Indeed, 1.8 and $1.5 \log$ increase in total microbial count was observed in the control and 1\% nano-silver groups after 24 months, respectively. Although a weak significant increase in total microbial count was observed in $2 \%$ nano-silver group, it was significantly lower than that of $1 \%$

A

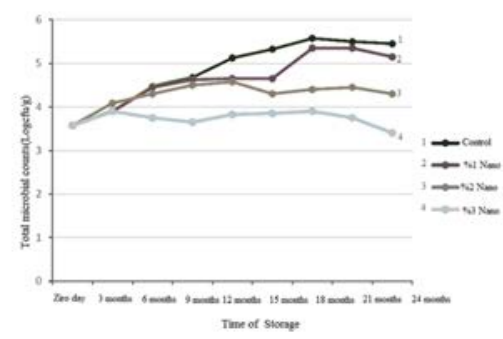

C

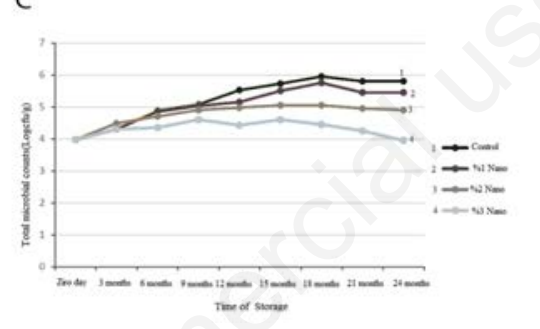

nano-silver and control groups. In general, the $3 \%$ nano-silver film inhibits microbial growth and at the end of the maintenance period, there was no increase in total count compared to the first day of study (Figure 1C). Similar results were obtained for packaged almond (Figrue 1D).

\section{Mold count}

Results of total mold count of packed nuts in different concentrations of nano-silver is depicted in Figure 2. In all studied

B

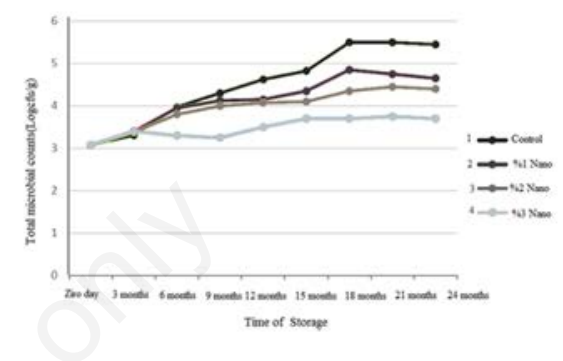

D

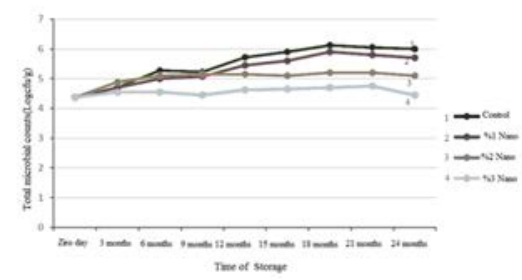

Figure 1. Total microbial count in nuts packed with films containing different percentages of nano-silver: walnut (A), hazelnut (B), pistachio (C), almond (D).

A

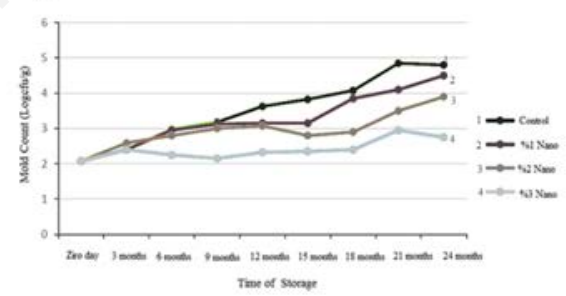

C

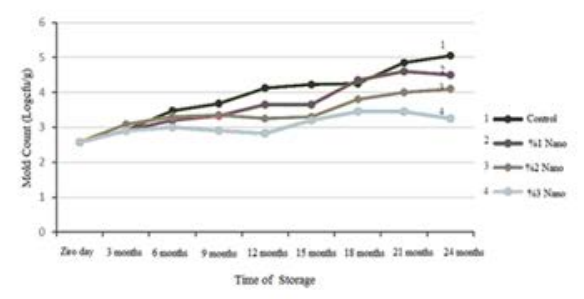

B

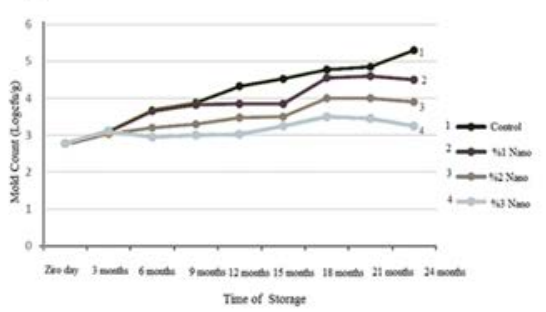

D

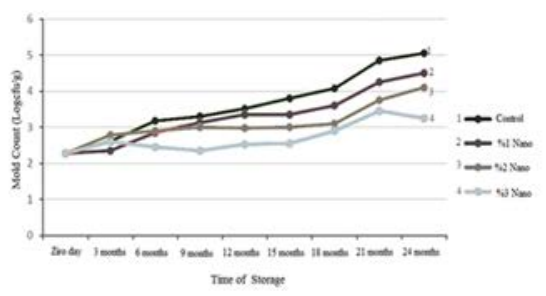

Figure 2. Mold count in nuts packed with films containing different percentages of nanosilver: walnut $(\mathrm{A})$, hazelnut $(\mathrm{B})$, almond $(\mathrm{C})$, pistachio (D). 
nuts mold count was increased in control group during the storage time. Nano-silver films reduced number of molds compared to control group; moreover, this inhibitory effect was increased in higher concentrations of nano-silver (Figure 2).

\section{Coliform count}

Results of total coliform count of packed nuts in different concentrations of nano-silver is depicted in Figure 3. In all nuts, one $\log$ increase in total coliform count was observed in the control group. Although coliform count was increased in 1 and $2 \%$ nano-silver groups, this increase was significantly lower than that in the control group. In 3\% nano-silver group, not only no increase in coliform count was observed, but also it was reduced in Pistachio (Figure 3D).

\section{Chemical analysis}

\section{Peroxide value}

Results of peroxide value of packed nuts in different concentrations of nano-silver are depicted in Figure 4. In all nuts, peroxide value was increased in the control group. In 1\% nano-silver group, peroxide value was not significantly different from the control group. In all studied nuts, increasing concentrations of nano-silver reduced peroxide value. While there were no difference in peroxide value between $2 \%$ and 3\% nano-silver groups in pistachio and almond, the 3\% nano-silver group had significantly a lower peroxide value compared to $2 \%$ nano-silver group in walnut and hazelnut.

\section{Aflatoxin detection}

Aflatoxin was not found in any of the nuts except walnut control group at 12th month and hazelnut and pistachios control groups at 24th month (Table 1). The detected aflatoxin in the samples of walnuts, hazelnuts and Pistachios were 2.1, 4.3, 2.3 $\mathrm{ng} / \mathrm{g}$ (ppb), respectively; that was lower than permissible deal, $10 \mathrm{ng} / \mathrm{kg}$ based on international Codex standards.

\section{Discussion}

The purpose of this study was to investigate the effect of nano-silver packaging in increasing the shelf life of packed nuts. Our results showed significant effect of using nano-silver in food packaging on preventing increase of microbial load and enhancing shelf life for four types of nuts including walnuts, hazelnuts, almonds and pistachios. While a gradual increase was observed in the total number of bacteria in the control group (nearly 2-3 log) during 24 months,

samples with 1, 2 and $3 \%$ concentration of nano-silver had significant decrease in total count of bacteria compared to the control group. Moreover, the reduction in 3\% nanosilver group, about $7 / 3 \log \left(5 \times 10^{3}\right)$, was much higher than other groups.

To our knowledge, no previous study has investigated efficacy of nano-silver particles in reducing the microbial load of nuts; however, several studies have proved the effect of nanoparticle coatings in reducing the number of bacteria in other foods. In a study conducted by the Ahari et al. (2013), effect of nano-silver on shelf life of Iranian saffron was investigated using nano-coatings SNP 103.3 and reported that 5\% nano-
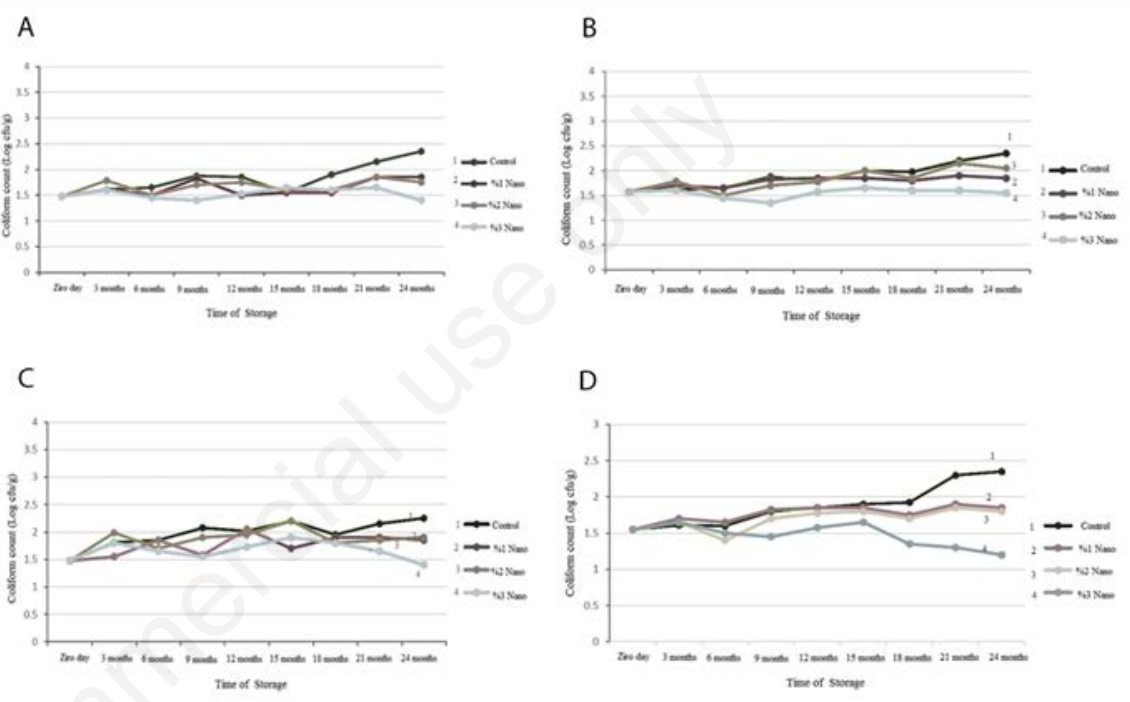

Figure 3. Choliform count in nuts packed with films containing different percentages of nano-silver: walnut (A), hazelnut (B), almond (C), pistachio (D).

silver, i.e. $4000 \mathrm{ppm}$, can reduce the microbial load by up to $98 \%$ (Ahari et al., 2013). Fernández et al. (2010), have investigated the efficacy of cellulosic pads containing silver nanoparticles in controlling the microbial flora (mesophilic aerobic psychotropic bacteria, mold and yeast) of packages containing a food model (Fernández et al., 2010). These pads increased the lag phase and reduced microbial load about 3 cycles compared to the control group. The results of this study is consistent with ours regarding its effect on microbial load. Incoronato et al. (2011) studied the effect of antimicrobial packaging system containing nano-silver on cheese quality and reported

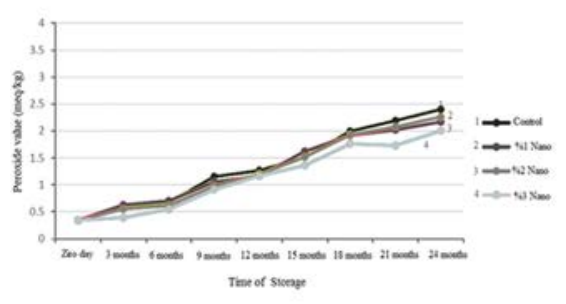

C

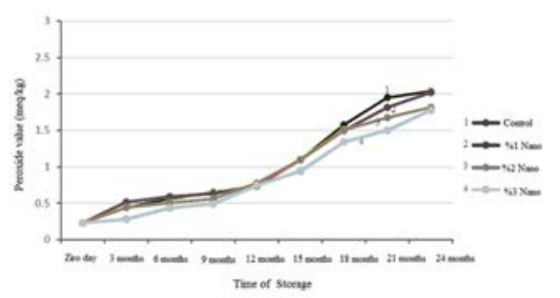

A

Figure 4. Peroxide value in nuts packed with films containing different percentages of nano-silver: walnut (A), hazelnut (B), almond (C), pistachio (D).
B

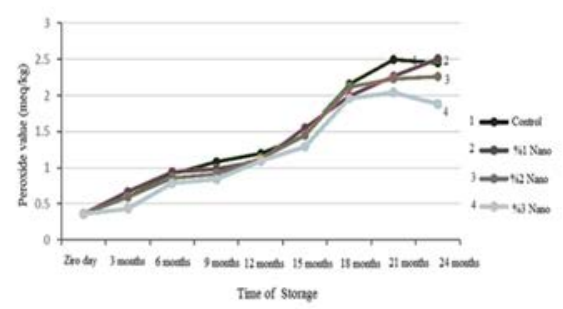

D

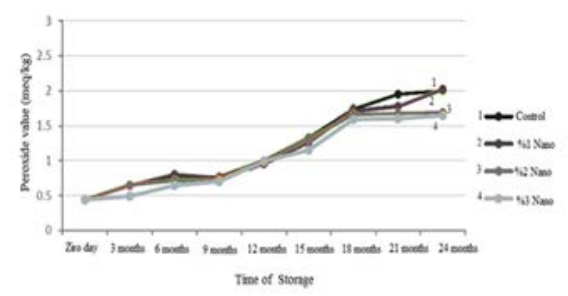


that while this packaging significantly increased shelf life of cheese, it had no undesirable effect on main germs, functional dairy and sensory characteristics of the product (Incoronato et al., 2011). Mahdi et al. (2012) investigated the effect of using nano-silver packaging in meat packing and reported that nano-silver reduced the microbial load and increased the shelf life about 2-7 days.

In this study, nano-silver packaging had a significant effect on the number of coliforms in the walnut, hazelnut, almond and pistachio compared to the control group; moreover, in 3\% nano-silver group, not even no increase was observed in the number of coliforms, but it was reduced in pistachio and in other nuts a significant decrease in the number of coliforms was observed compared with the control groups. Similar results were observed regarding the mold count of the packaged nuts. These results are consistent with previous studies that showed antifungal effects of nano-silver (Pulit et al., 2013; Mohammadi et al., 2014; Eslami et al., 2016). For instance, Mohammadi et al. (2014) investigated use of packaging containing 3-10\% nano-silver on the shelf life of bread and concluded that use of nano packaging effectively prevents mold growth during maintenance for 28 days and by increasing concentrations of nano-silver, this effect also increases (Mohammadi et al., 2014). Eslami et al. (2016) investigated effect of nano-silver packaging on shelf life of packaged saffron and found that adding $4000 \mathrm{ppm}$ nano-silver in the packing causes mold count to decrease to $4 \log$ during 6 months (Eslami et al., 2016); these results are consistent with those of ours which showed an average $3 \log$ reduction in mold count in most groups when $3 \%$ nano-silver was used. Furthermore, by decreasing mold count in treated samples, aflatoxin was not found in none of them in our study which is consistent with a previous study (Mousavi et al., 2016).

Regarding the peroxide value of packaged nuts, our results showed that it was increased in control group of all nuts during maintenance. For example, in the pistachios and almonds, it increased to $2 \mathrm{mg} / \mathrm{kg}$ and in hazelnut and walnut to $2 / 5 \mathrm{mg} / \mathrm{kg}$ after 24 months. Increasing concentrations of nanosilver caused a more reduction in peroxide value in all studied nuts. These effects were greater at $3 \%$ concentration of nano-silver and resulted a significant decrease in peroxide value especially in walnut and hazelnut. Greater effect of nano-silver on walnut and hazelnut may be attributable to their more fat-rich and antioxidant activity compared to almonds and pistachios. Mousavi et al.
(2016) showed antioxidant properties of 3 and 5\% nano-silver packaging and their beneficial effect on sensory and organoleptic quality of Mazafati date within 53 days of storage and also showed that the use of nanoparticles causes fixed physical features including color of fresh pistachio )Mousavi et al., 2016). The shelf life of nuts in different groups was determined based on the before-mentioned microbial and chemical analysis. Accordingly, our results showed that the highest shelf life belongs to pistachios, almonds, hazelnuts and walnuts with 20, 19, 18 and 18 months, respectively. The shelf life of control group in similar storage conditions were calculated for an average of 13 months. The highest shelf life in each nut was achieved by using 3\% nano-silver concentration. The lower shelf life of walnut compared to other nuts is likely attributable to its more fat-rich and $\mathrm{a}_{\mathrm{w}}$ (activated water). The higher shelf life of treated samples compared to control samples may be related to the fact that nanocomposite packaging prevents penetration of oxygen, carbon dioxide and humidity into the container and so increases durability of nutrients.

\section{Conclusions}

In summary, results of this study showed that using nano-silver significantly decreases molds, coliform and peroxide value in packaged samples. According to these results, longest shelf life obtained for pistachio, almond, hazelnut and walnut, respectively. While shelf life was 13 months for control sample, it was 20,19 and 18 months for above nuts, respectively, representing a significant increase in the shelf life of treatment groups compared to the control groups. However, our results should be interpreted in the light of some considerations. Although the ability of silver nanoparticles to migrate from the packaging material into the food and its effect on human health is controversial (Rajeshkumar and Malarkodi, 2014; Martirosyan and Schneider, 2014), the possible introduction of silver in nut samples was not investigated in this study. Hence, extensive research about application of nano-silver in packaging and its effect on human health is recommended. There is thus a current need to study the relationships between particle characteristics, polymer type, food $\mathrm{pH} /$ polarity and environmental conditions relevant to food production, storage and packaging (e.g., temperature, pressure, humidity, light exposure, storage time).

\section{References}

Ahari H, Anvar A, Shokri A, Bayat M, Talakesh F, Sadeghi M, Rahmannia H, 2013. Survey of shelf life effect on Iranian saffron with nano packaging SNP 103.3 for microbial properties and Nano particle release. J Compar Pathobiol Iran 9:793-802.

Bumbudsanpharoke N, Ko S, 2015. Nanofood packaging: an overview of market, migration research, and safety regulations. J Food Sci 80:R910-23.

Carbone M, Sabbatella G, Antonaroli S, Remita H, Orlando V, Biagioni S, Nucara A, 2016. Exogenous control over intracellular acidification: Enhancement via proton caged compounds coupled to gold nanoparticles and an alternative pathway with DMSO. Data Brief 6:745-9.

Costa C, Conte A, Buonocore G, Del Nobile M, 2011.Antimicrobial silver-montmorillonite nanoparticles to prolong the shelf life of fresh fruit salad. Int J Food Microbiol 148:164-7.

Darroudi M, Ahmad MB, Abdullah AH, Ibrahim NA, Shameli K, 2010. Effect of accelerator in green synthesis of silver nanoparticles. Int J Mol Sci 11:3898905.

Emamifar A, Kadivar M, Shahedi M, Soleimanian-Zad S, 2010. Evaluation of nanocomposite packaging containing $\mathrm{Ag}$ and $\mathrm{ZnO}$ on shelf life of fresh orange juice. Innov Food Sci Emerg Technol 11:742-8.

Eslami M, Bayat M, Nejad ASM, Sabokbar A, Anvar AA, 2016. Effect of polymer/nano-silver composite packaging on long-term microbiological status of Iranian saffron (Crocus sativus L.). Saudi J Biol Sci 23:341-7.

Fernández A, Picouet P, Lloret E, 2010. Cellulose-silver nanoparticle hybrid materials to control spoilage-related microflora in absorbent pads located in trays of fresh-cut melon. Int J Food Microbiol 142:222-8.

Foroughi S, Dabbagh MA, Ahari H, Akbarein H, Anvar A, Aghazadeh MM, Ghanbari SN, 2011. A Survey on the shelf life extension of foods with nanofilms. Ann Mil Health Sci Res 816.

Incoronato A, Conte A, Buonocore G, Del Nobile M, 2011.Agar hydrogel with silver nanoparticles to prolong the shelf life of Fior di Latte cheese. J Dairy Sci 94:1697-704.

Mahdi S, Vadood R, Nourdahr R, 2012. Study on the antimicrobial effect of nano-silver tray packaging of minced beef at refrigerator temperature. Glob 
Vet 9:284-9.

Martínez-Abad A, Lagaron JM, Ocio MJ, 2012. Development and characterization of silver-based antimicrobial ethylene-vinyl alcohol copolymer (EVOH) films for food-packaging applications. J Agric Food Chem 60:5350-9.

Martirosyan A, Schneider Y-J, 2014. Engineered nanomaterials in food: implications for food safety and consumer health. Int $\mathrm{J}$ Environ Res Public Health 11:5720-50.

Mohammadi H, Anvar A, Qajarbeygi P, Ahari H, Abdi F, 2014. Comparison of the antifungal activity of titanium dioxide based nano-silver packaging and conventional polyethylene packaging in consumed bread. App Food Biotechnol 2:45-51.
Morris Jr JG, 2011. How safe is our food? Emerg Infect Dis 17:126.

Mousavi FP, Pour HH, Amir Heidari Nasab AAR, Barouni M, 2016. Investigation Into Shelf Life of Fresh Dates and Pistachios in a Package Modified With Nano-Silver. Glob J Health Sci 8:134.

Pulit J, Banach M, Szczygłowska R, Bryk M, 2013. Nano-silver against fungi. Silver nanoparticles as an effective biocidal factor. Acta Biochim Pol 60:7958.

Rajeshkumar S, Malarkodi C, 2014. In vitro antibacterial activity and mechanism of silver nanoparticles against foodborne pathogens. Bioinorg Chem Appl 2014:1-10.

Rashidi L, Khosravi-Darani K, 2011. The applications of nanotechnology in food industry. Crit Rev Food Sci Nutr 51:723-30.

Sánchez-Valdes S, Ortega-Ortiz H, Ramosde Valle L, Medellín-Rodríguez F, Guedea-Miranda R, 2009. Mechanical and antimicrobial properties of multilayer films with a polyethylene/silver nanocomposite layer. J Appl Polym Sci 111:953-62.

Sattari M, Minaee S, Azizi M, Afshari H, 2010. Effects of nanofilms packaging on organoleptic and microbial properties of bread. Iran J Nut Sci Food Technol 4:65-74.

Toker R, Kayaman-Apohan N, Kahraman M, 2013. UV-curable nano-silver containing polyurethane based organicinorganic hybrid coatings. Prog Org Coat 76:1243-50. 